\title{
La educación supranacional, el rol de la universidad y la realidad del Chad: conectando lo global con la urgencia de lo local
}

\author{
Asenjo Gómez, Juan Tomás \\ Universidad Pontificia Comillas, Madrid, España \\ jtasenjo@comillas.edu
}

\section{Resumen}

La consolidada sociedad de la globalización y la interdependencia entre naciones ha desembocado en un renovado marco internacionalizado de educación, donde las metas de calidad y acreditación conviven con el reto de afrontar desequilibrios y desigualdades cada día más acentuados. En el ámbito educativo emergen con decisión las entidades internacionales; organizaciones que se han propuesto liderar proyectos educativos que ya hace tiempo superan los límites nacionales para instalarse en las necesidades internacionales. Es en esta parcela en la que la educación supranacional asume la valoración de las políticas educativos que acompañan a las instituciones internacionales. Al tiempo, la universidad que surge del Espacio Europeo de Educación Superior no puede eludir una misión conjugada en términos de Responsabilidad Social Universitaria y comprometida con la inclusión y la equidad de género en los países en vía de desarrollo. Una formación ética sustentada en el rol de profesor como agente de cambio social; y reflejada en el alumnado por medio del compromiso del Aprendizaje y Servicio universitario. A través del estudio de los documentos que emanan de las diferentes instituciones internacionales nos hemos propuesto analizar los convenios educativos internacionales establecidos con el continente africano, así como la actualidad de los diferentes programas universitarios de responsabilidad social de la Comunidad de Madrid y la concreción de acciones de Aprendizaje y Servicio universitario. Análisis implementado con el estudio de un caso, ubicado en un colegio de la República del Chad, en el que la colaboración internacional concurre con la inclusión educativa de las niñas y la formación de adultos. Pretendemos, para concluir, reflexionar cómo la convergencia entre continentes tendrá que cimentarse desde posibilitar el principio de Educación para Todos, comenzando por aquellos que históricamente han sido excluidos, y desde la construcción de un modelo educativo sostenible y de una ciudadanía comprometida con su futuro.

\section{Abstract}

The consolidation of the globalized society and the interdependence between nations has led to a renewed internationalized education framework, where the goals of quality and accreditation coexist with the challenge of facing imbalances and inequalities more and more accentuated. In the educational field, international entities emerge with decision; organizations that have the purpose to lead educational projects that have long exceeded national limits to be placed on international needs. It is in this area in which supranational education assumes the assessment of educational policies that accompany international institutions. At the same time, the university that emerges from the European Higher Education Area cannot avoid a conjugated mission in terms of University Social Responsibility and committed to inclusion and gender equity in the developing countries. An ethical education based on the role of teacher as an agent of social change; and reflected in the students through the commitment of the Learning and University Service. Along the study of the documents that emanate from the different international institutions we have proposed to analyze the international educational agreements established with the African continent, as well as the present of the different university programs of social responsibility of the Community of Madrid and the concretion of actions of Learning and university Service. Analysis implemented with the study of a case, located in a school in the Republic of Chad, in which the international collaboration concurs with the education inclusion of girls and the training of adults. We intend, in conclusion, to reflect on how the convergence between continents will have to be based on enabling the principle of Education for All, starting with those that historically have been excluded, and from the construction of a sustainable educational model and a citizenship committed to its future.

Palabras clave: supranacional, universidad, Chad, inclusión, Educación para Todos.

Keywords: supranational, university, Chad, inclusion, Education for All. 


\section{INTRODUCCIÓN}

La educación, tal y como la conocemos, se ha convertido en objeto de toda una dialéctica presente en foros hasta ahora impensables, pues se aborda desde los ámbitos económicos hasta los sociológicos y filosóficos. Esta revisión, que se suma al concepto de «repensar» pero que nos gusta creer que se aleja del de «rehacer» y de la educación líquida (Bauman, 2015), viene determinada por la era de la digitalización, la sociedad del conocimiento y la información, el capitalismo global, así como por la denominada internacionalización de la educación. En el centro de este nuevo horizonte se sitúa, como no había sucedido en épocas precedentes, al individuo en ese rol de ciudadano activo. Ya en el Tratado de Ámstedam de 1997 se formulaba la necesidad de impulsar una ciudadanía más activa y participativa en la vida de la comunidad. Una ciudadanía a la que Martínez (2004) sitúa en contextos de solidaridad, y sobre la que plantea la necesidad de ofertar «propuestas pedagógicas en las que se procuren a la vez hacer compatible la construcción de modelos de vida buena con modelos de vida justa» (p. 18).

El nuevo rol ciudadano se ve asociado con la obligación de asumir la responsabilidad de la educación -educación a lo largo de la vida-. Responsabilidad que tradicionalmente ha recaído en los gobiernos nacionales pero que a su vez se ve acompañada por diferentes organismos internacionales que, como argumenta Valle (2015), han promovido amplios proyectos de cooperación internacional en el área de la educación. Este nuevo contexto es fruto de una dinámica social que ha generado la consolidación de dichas instituciones ante la necesidad de modelos sociales más justos, democráticos y dignos. En medio de este panorama, aupado por la movilidad académica, surge la internacionalización de la educación, de forma especial en la educación superior. Lo que expone a la universidad ante el desafío de repensar la identidad de una institución en evidente fase de renovación. Una internacionalización que no es sólo educativa; el mundo interconectado establece una globalización económica, política, cultural, en tanto que nos muestra muchas zonas del planeta donde las urgencias de alimentos, sanidad y educación se acompañan de las carencias de justicia, derechos humanos y paz. Pero si hay un lugar en el que se suman todos estos males, y a gran escala, como las propias vivencias le llevan a afirmar a Bueno (2001), ese lugar se llama África. Nos enfrentamos, sin duda, al verdadero reto de la ciudadanía, de los gobiernos, del marco supranacional: dar respuestas de justicia y equidad, de humanidad, para el global de la población.

\section{LA ACCIÓN SOCIAL DESDE EL CONTEXTO SUPRANACIONAL}

El interés suscitado por la educación entre los estamentos no académicos está favoreciendo un protagonismo hasta ahora desconocido por el ámbito educativo. Buena parte de este impulso se encuentra ligado al último tercio del siglo pasado, concretado en la firme apuesta de organizaciones internacionales como la UNESCO, o incluso la OCDE. Este papel también toma forma en el marco de la Unión Europea, donde la Declaración de Bolonia supone algo más que una armonización de programas y títulos, y donde el Espacio Europeo de Educación imprime una acción educativa coordinada. Tendencias que se condensan en lo que Vega (2010) mantiene al afirmar que la educación debería contemplarse más desde el prisma mundial que desde identidades nacionales, señalando, junto a otros autores (Morvaridi, 2016; Sanahuja, 2007) la importancia de reflexionar sobre nuevos enfoques de actuación conjunta.

Los objetivos educativos marcados por las diferentes organizaciones, así como las numerosas formas de gestionar los proyectos pertenecientes al ámbito educativo nos dan una visión del interés real por participar en el progreso de un continente históricamente olvidado, África. Tras la Conferencia Mundial sobre Educación para Todos (Jomtien, Tailandia, 1990) la UNESCO aceptó la tarea de coordinación de este movimiento educativo. Entre los seis objetivos establecidos por la Conferencia se encuentran: Objetivo 2. Proporcionar enseñanza primaria gratuita y obligatoria para todos. Objetivo 4. Aumentar el número de adultos alfabetizados. Objetivo 5. Lograr la igualdad entre los géneros. Más tarde, la UNESCO asumirá el encargo de coordinar la Agenda de Educación Mundial 2030 en el marco de los Objetivos de Desarrollo Sostenible. Entre los objetivos que se fijaron se encuentran: Objetivo 4. Garantizar una educación inclusiva y equitativa de calidad y promover oportunidades de aprendizaje permanente para todos. Objetivo 5. Lograr la igualdad de género y empoderar a todas las mujeres y las niñas. Nos encontramos, a todas luces, ante unos retos que exigen toda la atención internacional; retos que se encuentran en la agenda de los diferentes organismos y gobiernos, pero sobre los que queda mucho trabajo de análisis, actuación y concienciación. 
Durante el año 2012, la entonces Directora General de la UNESCO, Irina Bokova, visitaba un país especialmente vulnerable, la República del Chad, donde recordaba los principios básicos de la actividad formativa promovida por la UNESCO: equidad, calidad y sostenibilidad. A la vez que marcaba los ámbitos que se reforzarían en el país visitado: la planificación y la gestión del sistema educativo, la alfabetización de adultos, la educación no formal, la renovación de los planes de estudios y la formación de docentes. Pero, a su vez, ponía el énfasis en una tarea pendiente y normalmente relegada, a saber, la necesidad de obtener datos estadísticos fiables en cuanto paso obligado a la hora de proyectar y estructurar una planificación eficaz.

Respecto a la Unión Europea, en la $5^{\text {a }}$ Cumbre Unión Africana-Unión Europea (UA-UE) ${ }^{1}$ que se celebró los días 29 y 30 de noviembre de 2017 en Abiyán (Costa de Marfil) se establecieron los siguientes cuatro ámbitos estratégicos: oportunidades económicas para la juventud, paz y seguridad, movilidad y migración, cooperación en materia de gobernanza. Hablar de África es hablar también de juventud, pues el $60 \%$ de la población africana es menor de 25 años. Por lo que a la citada Cumbre la acompañó de forma paralela la $4 .^{a}$ Cumbre de la Juventud África-Europa ${ }^{2}$, y el Plan de Acción Regional para el Sahel 2015-20203; en donde, de nuevo, se hace hincapié en crear condiciones adecuadas para la juventud a lo largo de toda esta región. Si para el Banco Mundial África es el principal destino en inversión, desde la Unión Europea se confirma que la suma de Estados miembros ocupan el primer puesto en cuanto a contribución en África: 21.000 millones de euros de ayuda para el desarrollo en 2016, lo que los sitúa como los mayores donantes de ayuda del continente; las empresas de la UE invirtieron 32.000 millones de euros en África en 2015, lo que equivale a alrededor de un tercio del total de la inversión extranjera directa en África; 1.400 millones de euros para programas educativos en África desde 2014 hasta 2020. Si nos centramos en uno de los países de la Región del Sahel, el Chad, entre 2008 y 2013 la financiación por parte del Fondo Europeo de Desarrollo (FED) fue por valor de 328 millones de euros, cantidad que ascendió a 442 millones en el $11^{\circ} \mathrm{FED}$. A partir de aquí, el Chad ha contado con un plan de desarrollo de cinco años, 2016-2020 bajo la denominación: «Le Tchad que nous voulons».

Otra línea de actuación es la del Banco Mundial, con una iniciativa clara: «Aprendizaje para todos». Una estrategia que se mueve bajo el lema: Invertir temprano. Invertir con inteligencia. Invertir para todos; cuyo objetivo es promover reformas a nivel nacional en los sistemas educativos, y cuya finalidad es impulsar acciones educativas que superen la etapa de la escolarización básica, alcanzando a toda la población. Un proyecto en el que se encuentra la Iniciativa Vía Rápida de Educación para Todos ${ }^{4}$, enfocada hacia aquellos países de ingreso bajo; se pretende, a través de donaciones de rápido desembolso, financiar procesos en línea con la consecución de los Objetivos de Desarrollo del Milenio (ODM) relativos a la educación.

Entre estos Objetivos se encuentra: Lograr la enseñanza primaria universal. Y como indican los datos, el África subsahariana presenta la mayor mejoría en enseñanza primaria respecto a otras regiones del mundo, logrando un aumento de 20 puntos porcentuales en la tasa neta de matriculación en el período de 2000 al 2015. En un claro contraste se encuentra el Chad, cuya tasa de alfabetización ${ }^{5}$, pasó del 25,264 en el año 2000, a caer a una tasa 22,312 en el año 2016, situándose en el segundo país con menor tasa de alfabetización del mundo (Tabla 1).

\footnotetext{
${ }^{1}$ Declaración conjunta de la 5a Cumbre Unión Africana-Unión Europea http://www.consilium.europa.eu/media/31991/33454-pr-final_declaration_au_eu_summit.pdf.

${ }^{2}$ Declaración de Abiyán https://www.africa-eu-partnership.org//sites/default/files/userfiles/4th_africa-europe_youth_summit_-_abidjan_declaration_2017.pdf.

${ }^{3}$ Plan de Acción Regional para el Sahel 2015-2020 http://www.consilium.europa.eu/media/21522/st07823-en15.pdf.

${ }^{4}$ Iniciativa Vía Rápida de Educación para Todos file://C:/Users/bragado\%20asenjo/Desktop/EFA-FTI_02\%2002\%202009_SP.pdf.

${ }^{5}$ Definición de alfabetización por la UNESCO: la aptitud para leer y escribir, comprendiéndolo, un texto sencillo y corto relacionado con la vida diaria.
} 
Tabla 1. Tasa de alfabetización.

\begin{tabular}{|c|c|c|c|}
\hline Fecha & País & Jóvenes (\%) & Adultos (\%) \\
\hline 2016 & Chad & 30,79 & 22,31 \\
\hline & España & 99,62 & 98,25 \\
\hline & Finlandia & 100 & 100 \\
\hline 2004 & Chad & 41,67 & 28,38 \\
\hline & España & 99,56 & 97,17 \\
\hline & Finlandia & 100 & 100 \\
\hline
\end{tabular}

Nota: Tomada de Expansión/Datos macro.

Los datos tampoco son prometedores en lo tocante al Índice de Brecha de Género, que analiza la división de los recursos y las oportunidades entre hombres y mujeres en 144 países, como puede verse en la Tabla 2.

Tabla 2. Índice Global de la Brecha de Género.

\begin{tabular}{|c|c|c|c|}
\hline Fecha & País & Ranking & Índice \\
\hline 2016 & Chad & 140 & 0,5867 \\
\hline & España & 29 & 0,7384 \\
\hline & Finlandia & 2 & 0,8450 \\
\hline 2006 & Chad & 113 & 0,5274 \\
\hline & España & 11 & 0,7345 \\
\hline & Finlandia & 3 & 0,795 \\
\hline
\end{tabular}

Nota: Tomada de Expansión/Datos macro.

\section{LA RESPONSABILIDAD SOCIAL UNIVERSITARIA}

El Comunicado que tuvo lugar tras la Conferencia Mundial sobre Educación celebrada en julio de 2009 en la sede de la UNESCO en París contempla que: los establecimientos de enseñanza superior del mundo entero tienen la responsabilidad social de contribuir a reducir la brecha en materia de desarrollo mediante el aumento de la transferencia de conocimientos a través de las fronteras, en particular hacia los países en desarrollo. Cometido que, acompañado por el valor asignado a la educación, por la tendencia al alza del interés hacia el área formativa, señala de manera directa a la misión desempeñada por la universidad en esta sociedad global del siglo XXI. La proyección social de las instituciones de educación superior, enmarcada en el fundamento de la Responsabilidad Social Universitaria, propone un nuevo eje de actividad del campus, alcanzando, como expone Vallaeys (2014), a toda la estructura universitaria. Un eje de actuación que conlleva la formación de personas comprometidas con su entorno, las cuales sean capaces de extender sus capacidades a las urgencias sociales asociadas a la evolución desigual de sociedades y países. Es desde este marco desde donde se impulsa la estrategia del Aprendizaje Servicio, entendida como una práctica reflexiva, actitudinal y de responsabilidad cívica del alumnado universitario. Y es aquí, también, donde la figura del profesor universitario adquiere un nuevo rol. Un profesor que, entendemos, precisa reafirmarse como verdadero agente social, de cambio social, aumentando sus competencias hacia los aspectos 
cívicos y sociales. Tal y como señala Core (2012), abarcando «la dimensión de los saberes (competencias culturales y didácticas), de los valores (responsabilidad educativa) y de la reflexión (conciencia y deontología profesional)» (p. 76). Labor que se concentraría en la formación de estudiantes con verdaderas actitudes de servicio hacia la comunidad, entendida ésta desde la globalidad solidaria. ¿De qué manera, nos preguntamos, se puede consolidar y fortalecer este aprendizaje en las nuevas generaciones? Propiciando, valoramos, la convivencia con el conocimiento; y, de manera simultánea, con las experiencias sociales desde esa propicia plataforma que supone las aulas universitarias. Por lo que si tuviéramos que definir el papel a desempeñar por las universidades se podría concretar en un compromiso académico con una sociedad inmersa en una dinámica global. De esta manera, se favorecería la vinculación del conocimiento de las realidades más necesitadas -aquellas realidades que se cruzan a nuestro paso, pero que también se vislumbran más allá de nuestras fronteras- con la experiencia de participar en la mejora de quienes carecen de lo más esencial. Esta propuesta educativa formaliza, como indica Domingo (2017), los cometidos de aprendizaje y de servicio; pero sólo si se incluye un tercer elemento: la transformación social. Lo que Deeley (2016) establece como «conducir al compromiso activo y cívico» (p. 167). Y Velasco (2008), acudiendo al prisma ético, puntualiza: «asociar lo que se estudia con la realidad, para hacernos conscientes de que algo, cada uno desde lo suyo, puede hacer, y todos juntos podemos algo más» (p.14). La Responsabilidad Social Universitaria ha alcanzado notoriedad suficiente como se ve reflejado en la abundante literatura que la acompaña (Puig, 2015; Battle, 2013; Naval, Ruiz- Corbellá, 2012; Arantzazu, Gómez, 2012). No obstante, como bien nos muestra Gaete (2010), el enfoque que se mantiene de manera decidida, aunque los itinerarios sean diferentes, está enfocado hacia esa necesaria transformación social (Tabla 3).

Es éste un modelo que, cimentado en las aulas universitarias, está calando en las estructuras académicas. Como ejemplo, el caso del ayuntamiento de Madrid, donde se ha firmado un convenio con los rectores de seis universidades madrileñas, Universidad Complutense, Universidad Politécnica, Universidad Autónoma, Universidad Juan Carlos I, Universidad Alcalá de Henares, Universidad Carlos III, más la UNED y la Universidad Menéndez Pelayo. Un convenio que propicia la implantación de la propuesta del Aprendizaje y Servicio universitario en torno a dos objetivos centrales: mejorar la calidad de vida de la ciudadanía y propiciar la inclusión social.

Tabla 3. Enfoques sobre la Responsabilidad Social Universitaria.

\begin{tabular}{|c|c|c|c|c|}
\hline $\begin{array}{l}\text { Perspectiva o } \\
\text { enfoque }\end{array}$ & \multicolumn{2}{|c|}{ Descripción } & $\begin{array}{l}\text { Algunos } \\
\text { Exponentes }\end{array}$ & Objetivo principal \\
\hline \multirow[t]{7}{*}{ Transformacional } & \multirow{7}{*}{$\begin{array}{l}\text { Se orientan } \\
\text { a revisar la } \\
\text { contribución } \\
\text { del quehacer } \\
\text { universitario } \\
\text { al necesario } \\
\text { debate y } \\
\text { reflexión para } \\
\text { alcanzar una } \\
\text { sociedad más } \\
\text { sustentable y } \\
\text { justa. }\end{array}$} & Formación & $\begin{array}{l}\text { Aprendizaje } \\
\text { servicio }\end{array}$ & $\begin{array}{l}\text { Formación de ciudadanos responsables, } \\
\text { transformación social. }\end{array}$ \\
\hline & & Investigación & $\begin{array}{l}\text { UNESCO } \\
(1998,2009)\end{array}$ & $\begin{array}{c}\text { Responsabilidad de la universidad en la } \\
\text { producción del conocimiento científico } \\
\text { demandado socialmente. }\end{array}$ \\
\hline & & & $\begin{array}{l}\text { Gibbons et ál. } \\
\qquad(1997)\end{array}$ & $\begin{array}{c}\text { Enfatiza la incorporación de múltiples actores } \\
\text { al proceso, y una conciencia social de los } \\
\text { problemas a investigar. }\end{array}$ \\
\hline & & Liderazgo & $\begin{array}{l}\text { Kliksberg } \\
\text { (2009) }\end{array}$ & $\begin{array}{l}\text { Liderazgo ético de la universidad, } \\
\text { participación en el debate de temas sociales. }\end{array}$ \\
\hline & & Social y & $\begin{array}{l}\text { Chomsky } \\
(2002)\end{array}$ & $\begin{array}{l}\text { Rol reflexivo, crítico y propositivo } \\
\text { sobre la sociedad. }\end{array}$ \\
\hline & & $\begin{array}{l}\text { Acción } \\
\text { Social }\end{array}$ & $\begin{array}{l}\text { Cooperación } \\
\text { universitaria } \\
\text { al desarrollo }\end{array}$ & $\begin{array}{l}\text { Transferir capacidades y conocimientos a } \\
\text { países en vías de desarrollo. }\end{array}$ \\
\hline & & & Multiversidad & $\begin{array}{l}\text { Respuestas académicas a las crecientes } \\
\text { expectativas sociales sobre su quehacer. }\end{array}$ \\
\hline
\end{tabular}




\section{CONCRETANDO LA ACCIÓN. UN COLEGIO EN LA REPÚBLICA DEL CHAD}

La República del Chad es un país situado en el corazón de África, comprendido en el denominado Sahel, auténtico cinturón del hambre y la pobreza, cuyas condiciones naturales semidesérticas y de inestabilidad política oprimen y asfixian al grupo de países que limitan al norte con el desierto del Sáhara y al sur con las sabanas y selvas del África Central, desde el Océano Atlántico hasta el mar Rojo. Hasta esta olvidada zona del mundo, en concreto hasta el colegio San Francisco Javier, situado a 20 kilómetros de la capital, Jamena, se ha desplazado un proyecto educativo de Aprendizaje y Servicio cuya puesta en marcha ha corrido a cargo de alumnos de educación y de ingeniería de la Universidad Pontificia Comillas. Los alumnos de ingeniería, con el propósito de construir un depósito de agua para los pocos pozos existentes gracias a la cooperación internacional. Los alumnos de educación, propiciando la misión de facilitar formación tanto en didácticas como en contenidos, desde las propias aulas del centro, así como en la tarea de la formación de profesores. El colegio en sí ya propone todo un proyecto de inclusión, a partir del momento en el que en las mismas aulas conviven diferentes culturas, etnias y religiones. Además, con una población estudiantil de casi un $40 \%$ de niñas, representa un esfuerzo por eliminar la discriminación de género. Un colegio que mantiene ese ideario de progreso que autores como Marchesi y Martí (2014), o Slee (2012) escenifican cuando sitúan a la escuela como plataforma de equilibrio, conocimiento y mejora social. En la misma línea, nos atrevemos a barajar cómo desde la conjugación del modelo educativo con el modelo social se propicia un avance en las oportunidades de desarrollo de las personas que habitan las zonas deprimidas coexistentes con el progreso. Al tiempo, la interactuación con este centro educativo chadiano posibilita que la población universitaria conozca de primera mano realidades que la globalización y los medios tecnológicos han convertido en cercanas a nivel de conocimiento, pero que continúan lejanas desde el ámbito de las actitudes; actitudes que se modifican, se dignifican, desde una alteridad vivida.

En este entorno de pobreza, UNICEF y los gobiernos de Camerún y Níger, con el apoyo de la Unión Europea, se ha acercado a la denominada «Crisis del Lago Chad». Se trata de un programa que busca dar apoyo a los 1,3 millones de niños que se han visto desplazados debido a la violencia causada por el grupo terrorista Boko Haram. Además, este programa ofrece una alternativa a los 200.000 niños que no pueden acceder a las escuelas en zonas afectadas por la crisis en la región de Diffa (Níger) y en el extremo norte de Camerún. El proyecto consiste en el desarrollo de un programa educativo utilizando como herramienta la radio. Ante las circunstancias del cierre de cientos de escuelas se ha desarrollado lo que denominan un prototipo regional de radio educativa que mantendrá a los niños en una rutina formativa, donde un programa de radio tiene la capacidad de alcanzar tanto a los niños que se encuentran en áreas inaccesibles a la ayuda humanitaria como a los niños cuyas escuelas han sido cerradas. A través de 144 episodios, con contenidos de lectura, escritura, matemáticas y mensajes de supervivencia y protección infantil, emitidos en francés y en las lenguas locales de Kanouri, Fulfulde y Hausa.

\section{DISCUSIÓN Y CONCLUSIONES}

La realidad de cifras abrumadoramente negativas, respecto a la satisfacción de una necesidad clave para el progreso personal y social como es la educación en África, exige medidas. La movilización internacional, por parte de los países denominados del primer mundo, en cuanto a la cristalización de recursos; y nacional, de los propios países en vías de desarrollo, en lo que respecta a la implementación de políticas educativas sostenibles. Europa, por su parte, necesita integrar en el discurso que acompaña a los programas y proyectos con el continente africano una prospectiva que vaya más allá de los aspectos mercantiles y profundice en las dinámicas sociales, culturales, educativas, ecológicas y de sostenibilidad. Porque sólo las acciones proactivas facilitarán la integración de África en las estructuras de valor internacionales. Cuenta para ello con el abrumador respaldo de la población, tal y como muestran las encuestas, en las que se muestra que casi nueve de cada diez ciudadanos de la UE apoyan la ayuda al desarrollo, con un 89\%, cuatro puntos porcentuales más que en 2014.

Con este marco, la responsabilidad social universitaria se traduce en la acción social desde el ámbito propio de un campus universitario, la producción de conocimiento. Pero un conocimiento que adquiere una doble entidad: por un lado, compartir ese conocimiento; por otro, contextualizar el conocimiento. Para finalizar con una elaboración conjunta y coordinada del conocimiento, propia del Aprendizaje y Servicio, y necesaria en las relaciones multilaterales de desarrollo. 
Son cuatro, entendemos, los fundamentos que estructuran la universidad del siglo XXI: la docencia y la investigación, como esencia de la actividad universitaria; la internacionalización como universalidad; y la responsabilidad social universitaria como compromiso social, como expresión del ámbito ético universitario.

\section{REFERENCIAS BIBLIOGRÁFICAS}

Battle, R. (2013). El aprendizaje servicio en España: el contagio de una revolución pedagógica necesaria. Madrid: PPC.

Bauman, Z. (2015). Los Retos De La Educación En La Modernidad Líquida. Barcelona: Gedisa. Bueno, A. (2001). Mirar a África. Redescubrir Europa. Barcelona: Cristianisme i justicia.

Comisión Europea (2014). Cooperación internacional y desarrollo. Luchar contra la pobreza en un mundo en transformación. Luxemburgo: Oficina de Publicaciones de la Unión Europea.

Core, P. (2012). ¿Resistir o innovar? La figura del docente entre crisis y relanzamiento de la formación. En J.C. Torre (Coord.), Educación y nuevas sociedades. Madrid: Universidad P. Comillas, ACISE-FIUC.

Deeley, S. (2016). El aprendizaje-servicio en educación superior. Teoría, práctica y perspectiva crítica. Madrid: Narcea.

Domingo, A. (2017). Conocimiento responsable y ciudadanía activa: las claves éticas del Aprendizaje-Servicio. En Martínez-Odria, A. y Gómez, I. (Coords.) (2017). Aprendizaje y Servicio. Educar para el encuentro. Madrid: Khaf.

Gaete, R. (2010). La responsabilidad social universitaria como desafío para la gestión estratégica de la Educación Superior: el caso de España. Revista de Educación, 355, 109-133.

Marchesi, Á. y Martín, E. (2014). Calidad de la enseñanza en tiempos de crisis. Madrid: Alianza.

Puig, J.M. (coord.). (2015).11 ideas clave ¿Cómo realizar un proyecto de Aprendizaje Servicio? Barcelona: Graó.

Slee, R. (2012). La escuela extraordinaria. Exclusión, escolarización y educación inclusiva. Madrid: Morata.

UNESCO (1998). La Educación superior en el siglo XXI: Visión y acción. Conferencia Mundial sobre la Educación Superior. París: UNESCO.

Vallaeys, Fr. (2014). La responsabilidad social universitaria: un nuevo modelo universitario contra la mercantilización. Revista Iberoamericana de Educación Superior, 5, (12), 105-117. 
Valle, J. M. (2015). Las políticas educativas en tiempos de globalización: la educación supranacional. Bordón, 67 (1), $11-21$. doi: 10.13042/Bordon.2015.67101

Vega, L. (2010). El proceso de Bolonia y la educación comparada. Miradas críticas. Salamanca: Ediciones Universidad de Salamanca. 